identity, is credited to Goodman (1991). However, Kurnit et al (1987) have argued previously that chance plays a major role in the occurrence of many developmental perturbations that cluster in families but recur less frequently than expected for simple Mendelian traits; these authors argue that for dysmorphic disorders showing non-Mendelian patterns of inheritance, randomness is intrinsic to morphogenesis such that a stochastic [probabilistic] single gene model can generate continuous liability curves very similar to those postulated by multifactorialpolygenic threshold models thereof. Given the weight of evidence that in schizophrenia the primary aetiopathophysiological process is one of disturbance(s) in early cerebral morphogenesis (Waddington, 1993), we have speculated that these formulations as to the role of chance in the genetic regulation of development may be relevant to the disorder (Waddington et al, 1994). Thus, to the extent that the entry of schizophrenia to the ranks of dysmorphic disorders is sustained, such theorising assumes a particular relevance for the continuing debate on the relative roles of 'genes $v$. environment' in its origins. There would still remain fundamental questions concerning not only the nature and timing of these early (genetically programmed, stochastic or other) dysmorphic events in schizophrenia but also the process(es) by which they might result in the evolution of psychotic symptoms and determine overall course of illness.

Goodman, R. (1991) Growing together and growing apart: the non-genetic forces on children in the same family. In The New Genetics of Mental Illness (eds P. McGuffin \& R. Murray), pp. 212-224. Oxford: Butterworth-Heinemann.

KURNIT, D. R., LAYTON, W. M. \& MATTHYSSE, S. (1987) Genetics, chance, and morphogenesis. American Journal of Human Genetics, 41, 979-995.

WADDINGTON, J. L. (1993) Schizophrenia: developmental neuroscience and pathobiology. Lancet, 341, 531-536.

-, O'Callaghan, E., Yousser, H. A., et al (1994) The neurodevelopmental basis to schizophrenia: beyond a hypothesis? In Schizophrenia-An Integrated View (Eds R. Fog, J. Gerlach, R. Hemmingsen, et al. Copenhagen: Munksgaard (in press).

Royal College of Surgeons in Ireland

JoHN L. WADDINGTON St. Stephen's Green

Dublin 2, Ireland

SIR: In his criticisms of our paper (McGuffin et al, $B J P$, May 1994, 164, 593-599), Eagles' (BJP, August 1994, 165, 266) comments show a profound misunderstanding both of what we were trying to say and of some of the papers he cites. Of course it is possible that twin studies do not on their own allow an accurate estimate of the magnitude of the genetic effect in schizophrenia, and that one possible source of error is that the prenatal environment is more similar in monozygotic (MZ) than dizygotic (DZ) pairs. However, as we pointed out, the evidence for an aetiological role for obstetric complications (whether pre-, peri- or post-natal) is extraordinarily difficult to interpret and suggests at most a very small effect. Indeed the incidence of schizophrenia is not markedly greater in twins in spite of their preponderance of early adverse events. Eagles describes us as using "clever formulae" to estimate heritability (with the implication that cleverness is somehow reprehensible). In fact the underlying principles are simple and straightforward with heritability estimated as double the difference between the $\mathrm{MZ}$ and $\mathrm{DZ}$ correlations.

Eagles goes on to quote the results of a family study by Kendler et al (1993) which he believes "used a preferable genetic method" (not, we think, a claim which Kendler and co-workers would make). He seems to believe that the results of this paper contradict our own findings. Kendler et al (1993) reported a comparatively low rate of schizophrenia in the parents of schizophrenics and described the likely explanation of this in terms of the diminished reproductive rates associated with schizophrenia. A more useful indicator of the familiarity of schizophrenia was that $9.2 \%$ of siblings of schizophrenics were affected compared with $0.5 \%$ of controls. This translates to a sibling correlation in liability of about $0.48( \pm 0.04)$. Since siblings share half of their genes, heritability can be roughly estimated by doubling the sibling correlation, i.e. a heritability of schizophrenia of about $96 \%$. Simply doubling the sibling correlation runs the risk of over-estimating heritability since this approach ignores non-additive genetic effects and shared environment. Nevertheless the results are not markedly different from our own estimate of a maximum heritability of $89 \%$.

Lastly, Eagles find it counter-intuitive that part of the aetiology of schizophrenia may be explained by random events. We described a number of genomic phenomena that can decrease the resemblance within pairs of relatives and gave examples of where these have been demonstrated in other diseases. Although strictly speaking not all such phenomena are stochastic they would all normally be interpreted as 'non-genetic' but yet would be completely undetectable by traditional epidemiological methods designed to investigate environmental factors in disease.

We are sorry that Eagles finds all this so perplexing and can only suggest that a read of a recent primer (e.g. McGuffin et al, 1994) will help remedy his present confusion. 
Kendler, K. S., MCGuire, M., Gruenberg, A. M., et al (1993) The Roscommon Family Study. I. Methods, diagnosis of probands and the risk of schizophrenia in relatives. Archives of General Psychiatry, 50, 527-540.

McGuffin, P., OWEn, M. J., O'Donovan, M. C., et al (eds) (1994) Seminars in Psychiatric Genetics. London: Gaskell.

Peter McGuffin

MiChAEL J. OWEN PHILIP ASHERSON ANNE E. FARMER

University of Wales College of Medicine Cardiff CF4 $4 X N$

\section{Cognitive-behavioural therapy for schizophrenia}

SIR: Patience (BJP, August 1994, 165, 266-267) comments that we overlook two problems in our Editorial (BJP, May 1994, 164, 581-587). He implies that we subscribe to "the notion that simply talking the patient out of delusions is of benefit" far from it, cognitive-behavioural treatment (CBT) of schizophrenia is much broader-based, involving techniques to tackle the range of psychopathology encountered (Kingdon \& Turkington, 1994). Delusions are viewed as neither 'core pathology' nor 'epiphenomena', but beliefs with meaning to the individual which deserve collaborative exploration and understanding.

Patience acknowledges that CBT is effective in depression and useful in other conditions. His statement that "simply using a treatment because it works can only be defended if at the same time efforts are made to understand why it works" is reasonable. Three-quarters of our article explored the phenomenology of psychosis, because we want to understand the nature of the disorder, to guide our interventions. One of us is directly researching psychotic thought processes using the cognitive psychology paradigms that he suggests (John \& Hemsley, 1992; John \& Dodgson, 1994). However, while the aetiology of schizophrenia remains controversial, and the mechanisms of action of interventions with proven efficacy, such as antipsychotic medication, unclear, there can be no justification for abandoning either these or newer interventions which show promise. Even where psychological symptoms are directly caused by organic impairments, for example head injury, patients can be taught cognitive strategies to compensate for functional deficits and reduce distress.

We therefore question Patience's assertion that we should not employ CBT in new areas because we do not yet understand exactly why it works and until the model of Beckian CBT for depression has been updated. This assertion seems to us an excellent example of the sort of faulty assumption, unsupported by evidence, which CBT has proved particularly effective at exposing and, after due collaborative attention to the evidence that is available, amending.

JoHN, C. H. \& DoDGson, G. (1994) Inductive reasoning in delusional thought. Journal of Mental Health, 3, 31-49.

- \& HemsLeY, D. R. (1992) Gestalt perception in schizophrenia. European Archives of Psychiatry and Clinical Neuroscience, 241, 215-221.

KIngdon, D. G. \& Turkington, D. (1994) Cognitive Behavioural Therapy for Schizophrenia. East Sussex: Lawrence Erlbaum.

Carolyn John

Hartlepool General Hospital

Newcastle NHS Trust

DOUGLAS TURKINGTON

\section{Bassetlaw NHS Trust}

DAVID Kingdon

SIR: The current view is still that "with psychotic disorders a cognitive theory of aetiology is not promoted" (BJP, July 1994, 165, 126-130). I believe I owe my full recovery 22 years ago from paranoid schizophrenia to being given a cognitive insight into the aetiology of my condition. At that time I heard voices and thought I was being controlled telepathically. I was told to think of my body as a car with two drivers, myself and the other half of my brain. My trouble was that although I used the other half, I never let it have a go at taking control. In return the other half of my brain made me hear voices and confused me in any way it could.

This therapeutic insight came from a fellow patient in a large mental hospital now closed down. I don't think my psychiatrist knew I had hallucinations - I did not tell him in case he extended my detention period or increased my medication.

Afterwards I gave an account of how cognitive insight had enabled my recovery (Rollin, 1980). I am not the only ex-psychotic sufferer who has used cognitive insight to effect a complete recovery and I know I am not the only one who has been asked to write an account afterwards.

I hope these accounts can be collated, and that it can be shown that giving sufferers from schizophrenia an understandable explanation of their condition does more good than harm.

Rolun, H. R. (1980) Coping with Schizophrenia, pp. 144-147. Andre Deutsch.

West Hall

JeFF THOMAS

Surrey TW9 $4 E E$ 